\title{
四种豆科作物的光合生理和生长发育对弱光的响应
}

\author{
邹长明 ${ }^{1}$ 王允青 ${ }^{2}$ 刘 英 ${ }^{2}$ 张晓红 唐 杉 $^{2}$ \\ ${ }^{1}$ 安徽科技学院, 安徽风阳 233100 ; $^{2}$ 安徽省农业科学院土壤肥料研究所, 合肥 230031
}

\begin{abstract}
摘 要 为了确定豆科作物作为林果行间套种作物的适宜性, 于2014年通过田间试验和盆栽试验对 4 种豆科作物的耐阴能力 及其光合生理进行了研究。测定全光和弱光处理(全光的 $48 \%$ )下豆科作物在初花期的叶片光合特征参数、光合色素含量与核 酮糖-1,5-二磷酸羧化酶(RuBPCase)活性, 监测其生长发育对弱光的响应。结果表明: 弱光使供试作物的最大净光合速率、光 饱和点、光补偿点、表观量子效率和暗呼吸速率不同程度地向耐阴植物变化; 弱光还使供试作物的净光合速率、气孔导度、 蒸腾速率、瞬时水分利用效率和RuBPCase活性不同程度地下降，而胞间 $\mathrm{CO}_{2}$ 浓度显著上升; 遮阴后，乌豇豆(Vigna cylindrica) 和 绿豆(Vigna radiata)的叶绿素(Chl) a 和 $\mathrm{Chl} b$ 含量显著增加, $\mathrm{Chl} \mathrm{a} / \mathrm{b}$ 值显著降低, 大猪屎豆(Crotalaria assamica)和望江南(Senna occidentalis) 的光合色素含量也有不同程度的变化; 弱光使供试作物茎变细, 侧枝数减少, 生物量和干物质积累效率降低, 根冠 比降低, 根瘤量减少, 叶片变小变薄, 叶片数减少, 叶面积指数降低; 弱光胁迫下, 大猪屎豆不开花, 望江南只开花不结实, 而 绿豆和乌豇豆开花数减少, 花期缩短, 种子产量显著降低。根据供试作物的光合特性和生长发育对弱光的响应, 得出它们的耐阴 能力排序是：乌豇豆>绿豆>望江南>大猪尿豆，其中乌豇豆和绿豆适宜与林果套种，而望江南和大猪屏豆不适宜套种。
\end{abstract}

关键词 豆科作物, 遮阴, 光合特性, 光合色素, 生长发育, 产量

引用格式: 邹长明, 王允青, 刘英, 张晓红, 唐杉 (2015). 四种豆科作物的光合生理和生长发育对弱光的响应. 植物生态学报, 39, 909-916. doi: $10.17521 /$ cjpe.2015.0087

\section{Responses of photosynthesis and growth to weak light regime in four legume species}

ZOU Chang-Ming ${ }^{1}$, WANG Yun-Qing ${ }^{2 *}$, LIU Ying ${ }^{2}$, ZHANG Xiao-Hong ${ }^{1}$, and TANG Shan ${ }^{2}$

${ }^{1}$ Anhui Science and Technology University, Fengyang, Anhui 233100, China; and ${ }^{2}$ Institute of Soils and Fertilizers, Anhui Academy of Agricultural Sciences, Hefei 230031, China

\section{Abstract}

Aims In order to determine the adaptability of legumes as the interplanting crops in fruit yards, field and pot experimental treatments with full natural light and weak light ( $48 \%$ of full natural light) regimes were conducted in 2014 to test the shade tolerance and physiological mechanisms of four legume species.

Methods The leaf photosynthetic characteristic parameters, photosynthetic pigments contents and the activities of ribulose-1,5-bisphosphate carboxylase (RuBPCase) were measured during the first bloom stage. The responses of growth to weak light were likewise studied.

Important findings The results showed that the maximum net photosynthetic rate, light saturation point, light compensation point, apparent quantum yield and dark respiration rate of the four legume species changed into those of shade-tolerant plants under the weak light stress. The weak light reduced the net photosynthetic rate, stomatal conductance, transpiration rate, water use efficiency and RuBPCase activity of the legumes. In contrast, the weak light increased intercellular $\mathrm{CO}_{2}$ concentration significantly. Contents of chlorophyll (Chl) a and $\mathrm{Chl} b$ in leaves of Vigna cylindrica (VCS) and Vigna radiata (VR) increased significantly, while $\mathrm{Chl}$ a/b in the leaves decreased dramatically after shading. Other two species changed photosynthetic pigments contents after shading. The weak light changed the growth of the four legume species, such as reducing stem diameter and branching quantity, reducing root nodule and root-shoot ratio, decreasing dry matter yield and dry matter accumulation efficiency, debasing leaf area and leaf thickness, reducing leaf quantity and leaf area index. Crotalaria assamica (CA) can not bloom under the weak light stress. Flowers were not strong enough to seed for Senna occidentalis (SO) under the weak light. The weak light also changed reproductive growth of VCS and VR significantly, such as debasing flowers quantity and flowering time, as well as decreasing seed yield. In conclusion, according to the

收稿日期Received: 2015-04-09 接受日期Accepted: 2015-07-23

* 通讯作者Author for correspondence (E-mail: yunqingw@126.com) 
responses of photosynthesis and growth to weak light regime in the legumes, we concluded that the shade tolerance ranking of four legume species from high to low is as follows: VCS, VR, SO and CA. VCS and VR are suitable for orchard interplanting. However, $\mathrm{SO}$ and $\mathrm{CA}$ are not suitable for orchard interplanting.

Key words legume, shading, photosynthetic characteristics, photosynthetic pigments, growth, yield

Citation: Zou CM, Wang YQ, Liu Y, Zhang XH, Tang S (2015). Responses of photosynthesis and growth to weak light regime in four legume species. Chinese Journal of Plant Ecology, 39, 909-916. doi: 10.17521/cjpe. 2015.0087

绿色植物积累的干物质有 $90 \%$ 以上来源于光合 产物, 光合作用决定了农业生产的前途(Knox et al., 2010)。因此, 光合生理的研究一直受到广泛的重 视。目前, 国内外学者已对花卉、果树、牧草、粮 食、园林植物及其他经济作物的光合特性进行了大 量研究, 发现光合作用不仅受温度、水分、养分、 空气、土壤等外界条件影响(刘玉梅等, 2010; 厉广 辉等, 2014; 武辉等, 2014; 张翠萍等, 2014), 而且受 光照和植物本身遗传特性所制约(Bloor \& Grubb, 2003; 陈显等, 2006; 姜武等, 2007; 祁娟等, 2013)。 资料显示, 弱光胁迫可改变植物根、茎及叶片形态 结构, 调节光合色素含量及光合酶活性, 影响植物 生长发育和干物质积累效率。遮阴后, 植物通常会 通过降低根冠比, 增大比叶面积, 增加叶绿素(Chl) 含量尤其是 $\mathrm{Chl} b$ 的含量, 降低光饱和点 $(L S P)$ 、光补 偿点 $(L C P)$ 和暗呼吸速率 $\left(R_{\mathrm{d}}\right)$, 提高表观量子效率 $(A Q Y)$ 等方式以适应弱光环境(陈煜等, 2006; 姜武 等, 2007; 张云等, 2014)。但不同植物种类因其本身 的遗传特性不同, 对弱光的生理响应有很大差异 (Bloor \& Grubb, 2003; 姜武等, 2007; 刘玉梅等, 2010; 祁娟等, 2013), 因此, 人们常通过盆栽试验 或田间试验来测定植物光合参数和光合色素含量以 比较或判断植物的耐阴能力。

间作套种是运用群落的空间结构原理, 以充分 利用空间和资源为目的而发展起来的一种立体农业 模式, 是我国传统农业的精华之一。据资料, 不同类 型作物间作套种可改善田间群体结构, 增强群体抗 逆性, 减少化肥、农药的施用量, 提高光能利用率、 资源利用率和土地产出率(Awal et al., 2006; Tanwar et al., 2014)。豆科作物因具有固氮能力和较强的耐 瘵能力, 被广泛用于和其他作物及经济林进行间作 套种, 研究表明, 豆科作物与禾本科作物的间作可 使豆科作物的固氮能力提高近 10 倍, 禾本科根系分 泌的麦根酸等植物铁载体可增加豆科作物对铁的吸 收, 而豆科作物分泌的有机酸又可以活化土壤磷素,
改善禾本科作物的缺磷症状( Li et al., 2003, 2004a, 2004b); 果园间作豆科绿肥能显著降低果园土壤温 度, 保持土壤水分, 提高土壤肥力 (温明霞等, 2011)。然而, 与单作相比, 在间作套种体系中的豆 科作物截获的光能较少, 弱光成为影响其生长发育 和产量的主要环境因子, 耐阴能力成为品种筛选的 重要指标。因此, 加强豆科作物耐阴性能及其机理 研究, 篎选耐阴能力强、适宜于间作套种的豆科作 物, 有利于促进立体农业的发展, 这对经济、社会与 环境具有重要意义。为此, 2014年选择4种豆科作物 在安徽省开展了耐阴能力及其机理研究, 从遮阴后 作物的光合特性变化、叶片光合色素含量与光合酶 活性变化、生长发育与形态建成, 以及鲜草产量、 干物质产量和种子产量等方面进行综合比较研 究, 为推广豆科作物与其他作物间作套种技术提供 依据。

\section{1 材料和方法}

\section{1 材料}

试验种子由国家种质资源库提供, 分别为绿豆 (Vigna radiate)、乌豇豆(Vigna cylindrica)、大猪屎豆 (Crotalaria assamica)和望江南(Senna occidentalis)。

\section{2 试验方法}

2014年4-10月于安徽风阳县府城镇山后街村绿 肥种质资源圃进行大田试验，每个小区面积 $12 \mathrm{~m}^{2}$, 分对照(全光照)和遮阴 2 个处理, 重复 3 次, 不施肥, 人工穴播, 行距 $50 \mathrm{~cm}$, 株距 $40 \mathrm{~cm}$, 每穴播种 3-4粒, 出苗后定植2株。遮阴处理用透光率约 $50 \%$ 的黑色遮 阳网架在 $1.5 \mathrm{~m}$ 高的竹桩上, 网四周边沿离地 $0.5 \mathrm{~m}$, 以利于通风, 用便携式光合仪测得正午透光率为 $(48 \pm 3) \%$, 分枝期开始遮阴直到成熟期结束。生长 期间观察调查生育期、株高、茎粗、分枝数、叶片 数、开花数、荚果数及其他生长发育特征。每小区 在初花期(10\%植株开花)收割 $6 \mathrm{~m}^{2}$ 植株(隔行割取地 上部), 在田间现场称取鲜质量后带回实验室用烘 
干法测定水分含量以得到干物质产量, 用干物质产 量除以生长天数得到干物质积累效率。初花期用便 携式叶面积仪测量叶片面积, 用游标卡尺测量叶片 厚度和茎粗。在成熟期分批收获荚果, 置于白色尼 龙网袋内暴晒, 充分干燥后分离出种子。同时按相 同处理方式进行盆栽试验，重复4次，每盆装砂-土 混合物 $4 \mathrm{~kg}$ (砂:土质量比为 $1: 1$ ), 不施肥, 每盆播种 10 粒, 出苗后留苗3株。在对照处理初花期, 用光合 仪于盆栽现场测定各处理作物叶片光合参数; 采集 鲜叶样品于实验室测定 $\mathrm{Chl}$ 与类胡萝卜素(Car)含 量、核酮糖-1,5-二磷酸羧化酶(RuBPCase)活性; 最 后破盆, 用水小心冲洗根部土壤, 洗净后测量地上 部和地下部鲜质量、干质量及根瘤鲜质量。

\section{3 测定指标及方法}

用LI-6400XT型便携式光合仪(LI-COR, Lincoln, USA), 于9:00-11:00测定各处理作物上部完全 伸展的功能叶的光合参数, 设定 $\mathrm{CO}_{2}$ 浓度为 380 $\mu \mathrm{mol} \cdot \mathrm{mol}^{-1}$ 、叶室温度 $25{ }^{\circ} \mathrm{C}$ 、相对湿度 $70 \%$, 光合有 效辐射 $(P A R)$ 梯度为 $1600,1400,1200,1000,800$, $600,400,200,100,50,20,0 \mu \mathrm{mol} \cdot \mathrm{m}^{-2} \cdot \mathrm{s}^{-1}$, 改变 $P A R$ 后稳定 $180 \mathrm{~s}$ 。以 $P A R$ 为 $x$ 轴, 净光合速率 $\left(P_{\mathrm{n}}\right)$ 为 $y$ 轴, 得到 $P_{\mathrm{n}}-P A R$ 响应曲线。用抛物线模型 $y=a x^{2}+b x+c$ 拟合该曲线(祁娟等, 2013), 其中抛物线顶点坐标 $y$ 值为最大净光合速率 $\left(P_{\mathrm{n} \text { max }}\right), 90 \% P_{\mathrm{n} \max }$ 下的对应 $x$ 值 为光饱和点 $(L S P)$; 同时用低 $P A R\left(0-200 \mu \mathrm{mol} \cdot \mathrm{m}^{-2}\right.$. $\mathrm{s}^{-1}$ )下的数据作直线回归方程 $y=a x+b$ (Harrison \& Platt, 1986; 祁娟等, 2013), $a$ 为表观量子效率 $(A Q Y)$, $-b$ 为暗呼吸速率 $\left(R_{\mathrm{d}}\right),-b / a$ 为光补偿点 $(L C P)$ 。测定气 体交换参数时选择 $P A R$ 为 $1000 \mu \mathrm{mol} \cdot \mathrm{m}^{-2} \cdot \mathrm{s}^{-1}$ 时的数 据, 包括 $P_{\mathrm{n}}$ 、气孔导度 $\left(G_{\mathrm{s}}\right)$ 、胞间 $\mathrm{CO}_{2}$ 浓度 $\left(C_{\mathrm{i}}\right)$ 和蒸腾 速率 $\left(T_{\mathrm{r}}\right)$, 用公式 $P_{\mathrm{n}} / T_{\mathrm{r}}$ 计算瞬时水分利用效率 $(W U E)$ 。 $\mathrm{Chl}$ 与 Car用 $95 \%$ 乙醇提取, 分光光度法 $665 \mathrm{~nm} 、 649$ $\mathrm{nm} 、 470 \mathrm{~nm}$ 下测定含量(王学奎, 2006); RuBPCase用 Tris-HCl缓冲液等提取, 分光光度法340 $\mathrm{nm}$ 下测定 活性(王学奎, 2006)。以上测定全部重复3次。

\section{4 数据分析}

测定结果用Excel 2003和SPSS 18.0软件进行分 析, 处理间比较用 $t$ 检验。

\section{2 结果和分析}

\section{1 遮阴后豆科作物光合特性变化}

用抛物线模型 $y=a x^{2}+b x+c$ 和直线回归方程 $y$ $=a x+b$ 拟合各处理作物 $P_{\mathrm{n}}-P A R$ 响应曲线后计算得 出的光合参数列于表 1 。结果显示, 遮阴后各处理作 物的 $P_{\mathrm{nmax}}$ 和 $L S P$ 均比对照显著下降, $L C P$ 除大猪屎 豆外都有显著降低, $R_{\mathrm{d}}$ 则是乌豇豆显著下降而其他 作物下降不明显; 遮阴后绿豆和乌豇豆的 $A Q Y$ 显著 提高，而大猪屎豆和望江南无明显变化。

\section{2 遮阴后豆科作物气孔特性和水分利用效率变化}

遮阴后 4 种豆科作物的 $P_{\mathrm{n}}$ 均显著下降, 同时还 伴随着 $G_{\mathrm{s}}$ 和 $T_{\mathrm{r}}$ 显著下降以及 $C_{\mathrm{i}}$ 的显著增加(表 2 )。另 外, 遮阴后多数作物的WUE也显著下降(唯乌豇豆 下降不显著), 作物在弱光下虽然减少了水分的蒸 腾损失 $\left(T_{\mathrm{r}}\right.$ 下降了 $\left.19 \%-32 \%\right)$, 但光合能力下降更多 $\left(P_{\mathrm{n}}\right.$ 下降 $\left.26 \%-50 \%\right)$, 因而 $W U E$ 下降了 7\%-37\%, $P_{\mathrm{n}}$ 与 $W U E$ 相关极显著, 相关系数 $r=0.8514(p<0.01)$ 。

\section{3 遮阴后豆科作物叶片中光合色素含量和光合 酶活性变化}

表3显示, 遮阴后, 供试作物的Chl a、Chl b和 Chl总量均有显著变化, 其中乌豇豆、绿豆和望江南 $\mathrm{Chl}$ 含量显著增加; 而大猪屎豆相反, 遮阴后 Chl含 量显著下降, 说明遮阴导致的光合产物供应不足妨 碍了 Chl的合成，这将影响其进一步的生长发育; Car含量在遮阴后有减少的趋势。供试作物在遮阴后 $\mathrm{Chl} \mathrm{a} / \mathrm{b}$ 变化趋势不一, 乌豇豆和绿豆显著减少, 望 江南无显著变化, 而大猪屎豆显著增加; 遮阴后各 种作物的RuBPCase活性均显著降低。

\section{4 遮阴后豆科作物营养生长状况}

遮阴对供试作物的营养生长产生了显著影响, 结果见表4和表 5 。

表4显示, 遮阴后, 供试作物茎粗显著变细, 侧 枝数显著减少, 大猪屎豆和望江南株高显著降低, 而绿豆和乌豇豆变化不显著(株高略有增加), 在地 下和地上生物量均显著降低的同时, 根/冠比也显 著减少。遮阴还严重抑制了根瘤的生长(根瘤鲜质量 仅为对照的 $1 / 8-1 / 6)$ 。

虽然遮阴对豆科作物地上部的影响不如地下部 严重, 但叶片性状和产量还是发生了很大的变化。 表 5 显示, 遮阴后, 供试作物的叶片数、单片叶面积、 叶面积指数、鲜草产量、干物质产量和干物质积累 效率均显著降低, 遮阴还导致作物叶片显著变薄。

\section{5 遮阴后豆科作物生殖生长状况及种子产量变化}

表 6 显示，遮阴导致绿豆提前 3 天开花，花期持 续天数缩短 9 天, 全生育期缩短 8 天, 开花数和结荚 
表1 盆栽豆科作物 $P_{\mathrm{n}}-P A R$ 响应曲线的特征参数(平均值士标准误差, $n=3$ )

Table 1 Characteristic values of $P_{\mathrm{n}}-P A R$ fit lines for the legumes in the pot experiment (mean $\pm S E, n=3$ )

\begin{tabular}{|c|c|c|c|c|c|c|c|}
\hline 种 Species & 处理 & Treatment & $\begin{array}{c}\text { 最大净光合速率 } P_{\text {max }} \\
\left(\mu \mathrm{mol} \mathrm{CO} \mathrm{CO}_{2} \cdot \mathrm{m}^{-2} \cdot \mathrm{s}^{-1}\right)\end{array}$ & $\begin{array}{l}\text { 光饱和点 } L S P \\
\left(\mu \mathrm{mol} \cdot \mathrm{m}^{-2} \cdot \mathrm{s}^{-1}\right)\end{array}$ & $\begin{array}{c}\text { 光补偿点 } L C P \\
\left(\mu \mathrm{mol} \cdot \mathrm{m}^{-2} \cdot \mathrm{s}^{-1}\right)\end{array}$ & $\begin{array}{c}\text { 表观量子效率 } \\
A Q Y\left(\mathrm{~mol} \cdot \mathrm{mol}^{-1}\right)\end{array}$ & $\begin{array}{c}\text { 暗呼吸速率 } R_{\mathrm{d}} \\
\left(\mu \mathrm{mol} \mathrm{CO} \mathrm{CO}_{2} \cdot \mathrm{m}^{-2} \cdot \mathrm{s}^{-1}\right)\end{array}$ \\
\hline \multirow{2}{*}{$\begin{array}{l}\text { 绿豆 } \\
\text { Vigna radiata }\end{array}$} & 对照 & $\mathrm{CK}$ & $21.09 \pm 0.51^{\mathrm{a}}$ & $1041 \pm 30^{\mathrm{a}}$ & $42.6 \pm 3.1^{\mathrm{a}}$ & $0.0251 \pm 0.012^{\mathrm{b}}$ & $1.069 \pm 0.078^{\mathrm{a}}$ \\
\hline & 遮阴 & Shading & $13.63 \pm 0.84^{\mathrm{b}}$ & $806 \pm 48^{b}$ & $30.9 \pm 2.9^{b}$ & $0.0314 \pm 0.011^{\mathrm{a}}$ & $0.969 \pm 0.073^{\mathrm{a}}$ \\
\hline \multirow{2}{*}{$\begin{array}{l}\text { 乌豇豆 } \\
\text { Vigna cylindrica }\end{array}$} & 对照 & $\mathrm{CK}$ & $25.52 \pm 0.33^{\mathrm{a}}$ & $1130 \pm 24^{\mathrm{a}}$ & $38.2 \pm 1.7^{\mathrm{a}}$ & $0.0313 \pm 0.015^{\mathrm{b}}$ & $1.196 \pm 0.052^{\mathrm{a}}$ \\
\hline & 遮阴 & Shading & $19.52 \pm 0.45^{\mathrm{b}}$ & $958 \pm 37^{\mathrm{b}}$ & $23.7 \pm 1.3^{\mathrm{b}}$ & $0.0384 \pm 0.009^{\mathrm{a}}$ & $0.911 \pm 0.047^{b}$ \\
\hline \multirow{2}{*}{$\begin{array}{l}\text { 大猪屎豆 } \\
\text { Crotalaria assamica }\end{array}$} & 对照 & CK & $24.36 \pm 0.41^{\mathrm{a}}$ & $1116 \pm 41^{\mathrm{a}}$ & $49.2 \pm 3.2^{\mathrm{a}}$ & $0.0265 \pm 0.013^{\mathrm{a}}$ & $1.304 \pm 0.065^{\mathrm{a}}$ \\
\hline & 遮阴 & Shading & $11.46 \pm 0.93^{\mathrm{b}}$ & $761 \pm 66^{b}$ & $43.8 \pm 3.4^{\mathrm{a}}$ & $0.0282 \pm 0.015^{\mathrm{a}}$ & $1.236 \pm 0.081^{\mathrm{a}}$ \\
\hline \multirow{2}{*}{$\begin{array}{l}\text { 望江南 } \\
\text { Senna occidentalis }\end{array}$} & 对照 & $\mathrm{CK}$ & $21.87 \pm 0.42^{\mathrm{a}}$ & $1113 \pm 39^{\mathrm{a}}$ & $46.9 \pm 0.5^{\mathrm{a}}$ & $0.0271 \pm 0.005^{\mathrm{a}}$ & $1.272 \pm 0.069^{\mathrm{a}}$ \\
\hline & 遮阴 & Shading & $11.75 \pm 0.59^{\mathrm{b}}$ & $767 \pm 74^{b}$ & $40.8 \pm 1.8^{\mathrm{b}}$ & $0.0284 \pm 0.023^{\mathrm{a}}$ & $1.158 \pm 0.111^{\mathrm{a}}$ \\
\hline
\end{tabular}

数据后不同字母表示同一作物不同处理间差异显著 $(p<0.05)$ 。

Different letters denote significant differences between treatments $(p<0.05)$. AQY, apparent quantum yield; $L C P$, light compensation point; $L S P$, light saturation point; $P_{\mathrm{nmax}}$, maximum net photosynthetic rate; $R_{\mathrm{d}}$, dark respiration rate.

表2 盆栽豆科作物的气体交换参数(平均值土标准误差, $n=3$ )

Table 2 Gas exchange parameters of the legumes in the pot experiment (mean $\pm S E, n=3$ )

\begin{tabular}{|c|c|c|c|c|c|c|}
\hline $\begin{array}{l}\text { 种 } \\
\text { Species }\end{array}$ & $\begin{array}{l}\text { 处理 } \\
\text { Treatment }\end{array}$ & $\begin{array}{c}\text { 净光合速率 } P_{\mathrm{n}} \\
\left(\mu \mathrm{mol} \mathrm{CO} \mathrm{CO}_{2} \cdot \mathrm{m}^{-2} \cdot \mathrm{s}^{-1}\right)\end{array}$ & $\begin{array}{c}\text { 气孔导度 } G_{\mathrm{s}} \\
\left(\mathrm{mol} \mathrm{H} \mathrm{H}_{2} \mathrm{O} \cdot \mathrm{m}^{-2} \cdot \mathrm{s}^{-1}\right)\end{array}$ & $\begin{array}{l}\text { 胞间 } \mathrm{CO}_{2} \text { 浓度 } C_{\mathrm{i}} \\
\left(\mu \mathrm{mol} \mathrm{CO}{ }_{2} \cdot \mathrm{mol}^{-1}\right)\end{array}$ & $\begin{array}{c}\text { 蒸腾速率 } T_{\mathrm{r}} \\
\left(\mu \mathrm{mol} \mathrm{H}_{2} \mathrm{O} \cdot \mathrm{m}^{-2} \cdot \mathrm{s}^{-1}\right)\end{array}$ & $\begin{array}{c}\text { 水分利用效率 } \\
W U E\left(\mathrm{~mol} \cdot \mathrm{mol}^{-1}\right)\end{array}$ \\
\hline \multirow{2}{*}{$\begin{array}{l}\text { 绿豆 } \\
\text { Vigna radiata }\end{array}$} & 对照 CK & $17.55 \pm 0.56^{\mathrm{a}}$ & $0.221 \pm 0.017^{\mathrm{a}}$ & $222.9 \pm 5.7^{b}$ & $3.98 \pm 0.18^{\mathrm{a}}$ & $4.41 \pm 0.18^{\mathrm{a}}$ \\
\hline & 遮阴 Shading & $10.12 \pm 0.33^{b}$ & $0.138 \pm 0.011^{\mathrm{b}}$ & $244.4 \pm 5.1^{\mathrm{a}}$ & $2.73 \pm 0.12^{b}$ & $3.71 \pm 0.14^{\mathrm{b}}$ \\
\hline \multirow{2}{*}{$\begin{array}{l}\text { 乌豇豆 } \\
\text { Vigna cylindrica }\end{array}$} & 对照 CK & $19.16 \pm 0.25^{\mathrm{a}}$ & $0.257 \pm 0.015^{\mathrm{a}}$ & $194.0 \pm 4.2^{\mathrm{b}}$ & $4.45 \pm 0.08^{\mathrm{a}}$ & $4.31 \pm 0.21^{\mathrm{a}}$ \\
\hline & 遮阴 Shading & $14.11 \pm 0.33^{\mathrm{b}}$ & $0.203 \pm 0.005^{\mathrm{b}}$ & $224.6 \pm 2.1^{\mathrm{a}}$ & $3.52 \pm 0.05^{\mathrm{b}}$ & $4.01 \pm 0.20^{\mathrm{a}}$ \\
\hline \multirow{2}{*}{$\begin{array}{l}\text { 大猪屎豆 } \\
\text { Crotalaria assamica }\end{array}$} & 对照 CK & $20.26 \pm 0.70^{\mathrm{a}}$ & $0.328 \pm 0.023^{\mathrm{a}}$ & $188.7 \pm 4.8^{\mathrm{b}}$ & $4.66 \pm 0.17^{\mathrm{a}}$ & $4.35 \pm 0.36^{\mathrm{a}}$ \\
\hline & 遮阴 Shading & $10.24 \pm 0.34^{\mathrm{b}}$ & $0.205 \pm 0.018^{\mathrm{b}}$ & $222.6 \pm 9.3^{\mathrm{a}}$ & $3.74 \pm 0.19^{\mathrm{b}}$ & $2.74 \pm 0.29^{b}$ \\
\hline \multirow{2}{*}{$\begin{array}{l}\text { 望江南 } \\
\text { Senna occidentalis }\end{array}$} & 对照 CK & $17.72 \pm 0.39^{\mathrm{a}}$ & $0.228 \pm 0.012^{\mathrm{a}}$ & $201.9 \pm 7.4^{b}$ & $4.24 \pm 0.21^{\mathrm{a}}$ & $4.18 \pm 0.22^{\mathrm{a}}$ \\
\hline & 遮阴 Shading & $10.85 \pm 0.43^{b}$ & $0.158 \pm 0.006^{\mathrm{b}}$ & $234.1 \pm 3.9^{\mathrm{a}}$ & $3.15 \pm 0.14^{b}$ & $3.44 \pm 0.13^{\mathrm{b}}$ \\
\hline
\end{tabular}

数据后不同字母表示同一作物不同处理间差异显著 $(p<0.05)$ 。

Different letters denote significant differences between treatments $(p<0.05)$. $C_{\mathrm{i}}$, intercellular $\mathrm{CO}_{2}$ concentration; $G_{\mathrm{s}}$, stomatal conductance; $P_{\mathrm{n}}$, net photosynthetic rate; $T_{\mathrm{r}}$, transpiration rate; $W U E$, water use efficiency.

表3 盆栽豆科作物鲜叶中光合色素含量及核酮糖-1, 5-二磷酸羧化酶(RuBPCase)活性(平均值土标准误差, $n=3$ )

Table 3 Content of photosynthetic pigments and activities of ribulose-1, 5-bisphosphate carboxylase (RuBPCase) in fresh leaves of the legumes in the pot experiment (mean $\pm S E, n=3)$

\begin{tabular}{|c|c|c|c|c|c|c|c|}
\hline $\begin{array}{l}\text { 种 } \\
\text { Species }\end{array}$ & $\begin{array}{l}\text { 处理 } \\
\text { Treatment }\end{array}$ & $\begin{array}{c}\text { 叶绿素a } \\
\mathrm{Chl} \mathrm{a}\left(\mathrm{mg}^{-1} \mathrm{~g}^{-1}\right)\end{array}$ & $\begin{array}{c}\text { 叶绿素b } \\
\mathrm{Chl} \mathrm{b}\left(\mathrm{mg} \cdot \mathrm{g}^{-1}\right)\end{array}$ & $\begin{array}{c}\text { 叶绿素总量 } \\
\mathrm{Chl}(\mathrm{a}+\mathrm{b})\left(\mathrm{mg} \cdot \mathrm{g}^{-1}\right)\end{array}$ & $\begin{array}{l}\text { 类胡萝卜素 } \\
\mathrm{Car}\left(\mathrm{mg} \cdot \mathrm{g}^{-1}\right)\end{array}$ & $\mathrm{Chl} \mathrm{a} / \mathrm{b}$ & $\begin{array}{c}\text { RuBPCase活性 } \\
\text { RuBPCase activity } \\
\left(\mu \mathrm{mol} \cdot \mathrm{g}^{-1} \cdot \mathrm{min}^{-1}\right)\end{array}$ \\
\hline \multirow{2}{*}{$\begin{array}{l}\text { 绿豆 } \\
\text { Vigna radiata }\end{array}$} & 对照 CK & $1.87 \pm 0.14^{b}$ & $0.40 \pm 0.12^{b}$ & $2.27 \pm 0.26^{\mathrm{b}}$ & $0.42 \pm 0.05^{\mathrm{a}}$ & $4.68 \pm 0.55^{\mathrm{a}}$ & $45.6 \pm 2.2^{\mathrm{a}}$ \\
\hline & 遮阴 Shading & $3.16 \pm 0.17^{\mathrm{a}}$ & $1.74 \pm 0.23^{\mathrm{a}}$ & $4.90 \pm 0.35^{\mathrm{a}}$ & $0.42 \pm 0.10^{\mathrm{a}}$ & $1.82 \pm 0.35^{\mathrm{b}}$ & $36.7 \pm 1.6^{\mathrm{b}}$ \\
\hline \multirow{2}{*}{$\begin{array}{l}\text { 乌豇豆 } \\
\text { Vigna cylindrica }\end{array}$} & 对照 CK & $1.83 \pm 0.02^{b}$ & $0.35 \pm 0.01^{\mathrm{b}}$ & $2.18 \pm 0.03^{\mathrm{b}}$ & $0.58 \pm 0.02^{\mathrm{a}}$ & $5.23 \pm 0.47^{\mathrm{a}}$ & $48.3 \pm 1.9^{\mathrm{a}}$ \\
\hline & 遮阴 Shading & $3.38 \pm 0.20^{\mathrm{a}}$ & $2.14 \pm 0.25^{\mathrm{a}}$ & $5.53 \pm 0.54^{\mathrm{a}}$ & $0.37 \pm 0.08^{b}$ & $1.58 \pm 0.32^{b}$ & $40.2 \pm 1.3^{b}$ \\
\hline \multirow{2}{*}{$\begin{array}{l}\text { 大猪屎豆 } \\
\text { Crotalaria assamica }\end{array}$} & 对照 CK & $2.19 \pm 0.08^{\mathrm{a}}$ & $0.63 \pm 0.08^{\mathrm{a}}$ & $2.82 \pm 0.16^{\mathrm{a}}$ & $0.75 \pm 0.14^{\mathrm{a}}$ & $3.50 \pm 0.23^{\mathrm{b}}$ & $52.8 \pm 1.7^{\mathrm{a}}$ \\
\hline & 遮阴 Shading & $1.59 \pm 0.14^{b}$ & $0.34 \pm 0.05^{\mathrm{b}}$ & $1.93 \pm 0.23^{b}$ & $0.59 \pm 0.04^{\mathrm{a}}$ & $4.68 \pm 0.31^{\mathrm{a}}$ & $23.5 \pm 3.1^{\mathrm{b}}$ \\
\hline \multirow{2}{*}{$\begin{array}{l}\text { 望江南 } \\
\text { Senna occidentalis }\end{array}$} & 对照 CK & $1.12 \pm 0.08^{\mathrm{b}}$ & $0.28 \pm 0.04^{\mathrm{b}}$ & $1.40 \pm 0.12^{\mathrm{b}}$ & $0.51 \pm 0.06^{\mathrm{a}}$ & $4.00 \pm 0.38^{\mathrm{a}}$ & $50.1 \pm 1.5^{\mathrm{a}}$ \\
\hline & 遮阴 Shading & $2.08 \pm 0.11^{\mathrm{a}}$ & $0.54 \pm 0.09^{\mathrm{a}}$ & $2.62 \pm 0.20^{\mathrm{a}}$ & $0.54 \pm 0.11^{\mathrm{a}}$ & $3.84 \pm 0.49^{\mathrm{a}}$ & $31.4 \pm 2.3^{\mathrm{b}}$ \\
\hline
\end{tabular}

数据后不同字母表示同一作物不同处理间差异显著 $(p<0.05)$ 。

Different letters denote significant differences between treatments $(p<0.05)$. Car, carotenoid; Chl, chlorophyll.

数减少至对照的 $1 / 5-1 / 4$, 英长、千粒重和种子产量 也显著降低(种子产量降为对照的 $1 / 6)$; 遮阴后，乌 豇豆的花期持续天数缩短 4 天, 而初花期和全生育
期无显著变化, 开花数和结英数减少至 $1 / 3-1 / 4$, 英 长、千粒重和种子产量也显著降低(种子产量降为对 照的 $1 / 5)$; 大猪屎豆在遮阴后生殖生长受阻, 不能

www.plant-ecology.com 
表4 遮阴对盆栽豆科作物根茎性状和根瘤的影响(平均值土标准误差, $n=3$ )

Table 4 Effect of shading on roots, stem and root nodules of the legumes in the pot experiment $(\operatorname{mean} \pm S E, n=3)$

\begin{tabular}{|c|c|c|c|c|c|c|c|c|}
\hline $\begin{array}{l}\text { 种 } \\
\text { Species }\end{array}$ & $\begin{array}{l}\text { 处理 } \\
\text { Treatment }\end{array}$ & $\begin{array}{c}\text { 株高 } \\
\text { Plant height } \\
(\mathrm{cm})\end{array}$ & $\begin{array}{c}\text { 茎粗 } \\
\text { Stem diameter } \\
(\mathrm{cm})\end{array}$ & $\begin{array}{c}\text { 侧枝数 } \\
\text { Branching } \\
\text { quantity }\end{array}$ & $\begin{array}{l}\text { 地下部鲜质量 } \\
\text { Fresh mass un- } \\
\text { derground part } \\
\left(\mathrm{g} \cdot \operatorname{pot}^{-1}\right)\end{array}$ & $\begin{array}{c}\text { 地上部鲜质量 } \\
\text { Fresh mass } \\
\text { overground part } \\
\left(\mathrm{g} \cdot \mathrm{pot}^{-1}\right)\end{array}$ & $\begin{array}{l}\text { 根/冠比 } \\
\text { Root-shoot } \\
\text { ratio }\end{array}$ & $\begin{array}{c}\text { 每盆根瘤质量 } \\
\text { Mass of root } \\
\text { nodule per pot } \\
(\mathrm{g})\end{array}$ \\
\hline \multirow{2}{*}{$\begin{array}{l}\text { 绿豆 } \\
\text { Vigna radiata }\end{array}$} & 对照 CK & $37.4 \pm 1.8^{\mathrm{a}}$ & $0.62 \pm 0.03^{\mathrm{a}}$ & $7 \pm 1^{\mathrm{a}}$ & $22.7 \pm 1.6^{\mathrm{a}}$ & $116.2 \pm 9.1^{\mathrm{a}}$ & $0.195 \pm 0.013^{\mathrm{a}}$ & $3.43 \pm 0.28^{\mathrm{a}}$ \\
\hline & 遮阴 Shading & $39.6 \pm 2.0^{\mathrm{a}}$ & $0.41 \pm 0.04^{\mathrm{b}}$ & $4 \pm 0^{\mathrm{b}}$ & $10.9 \pm 1.0^{\mathrm{b}}$ & $82.1 \pm 5.3^{\mathrm{b}}$ & $0.133 \pm 0.009^{\mathrm{b}}$ & $0.39 \pm 0.09^{\mathrm{b}}$ \\
\hline \multirow{2}{*}{$\begin{array}{l}\text { 乌豇豆 } \\
\text { Vigna cylindrica }\end{array}$} & 对照 CK & $45.7 \pm 2.1^{\mathrm{a}}$ & $0.75 \pm 0.06^{\mathrm{a}}$ & $8 \pm 1^{\mathrm{a}}$ & $29.6 \pm 1.1^{\mathrm{a}}$ & $144.8 \pm 8.2^{\mathrm{a}}$ & $0.204 \pm 0.017^{\mathrm{a}}$ & $2.76 \pm 0.17^{\mathrm{a}}$ \\
\hline & 遮阴 Shading & $48.3 \pm 2.6^{\mathrm{a}}$ & $0.48 \pm 0.02^{\mathrm{b}}$ & $5 \pm 0^{\mathrm{b}}$ & $14.8 \pm 0.8^{\mathrm{b}}$ & $108.1 \pm 4.7^{b}$ & $0.137 \pm 0.008^{\mathrm{b}}$ & $0.47 \pm 0.06^{\mathrm{b}}$ \\
\hline \multirow{2}{*}{$\begin{array}{l}\text { 大猪屎豆 } \\
\text { Crotalaria assamica }\end{array}$} & 对照 CK & $67.7 \pm 3.3^{\mathrm{a}}$ & $0.88 \pm 0.05^{\mathrm{a}}$ & $19 \pm 3^{\mathrm{a}}$ & $34.9 \pm 1.3^{\mathrm{a}}$ & $173.7 \pm 9.4^{\mathrm{a}}$ & $0.201 \pm 0.015^{\mathrm{a}}$ & $1.94 \pm 0.19^{\mathrm{a}}$ \\
\hline & 遮阴 Shading & $41.3 \pm 1.9^{\mathrm{b}}$ & $0.33 \pm 0.03^{b}$ & $5 \pm 1^{b}$ & $6.3 \pm 0.7^{b}$ & $52.0 \pm 4.5^{\mathrm{b}}$ & $0.121 \pm 0.007^{\mathrm{b}}$ & $0.25 \pm 0.04^{\mathrm{b}}$ \\
\hline \multirow{2}{*}{$\begin{array}{l}\text { 望江南 } \\
\text { Senna occidentalis }\end{array}$} & 对照 CK & $63.5 \pm 2.0^{\mathrm{a}}$ & $1.00 \pm 0.08^{\mathrm{a}}$ & $15 \pm 1^{\mathrm{a}}$ & $38.3 \pm 1.4^{\mathrm{a}}$ & $192.7 \pm 9.8^{\mathrm{a}}$ & $0.199 \pm 0.012^{\mathrm{a}}$ & $2.39 \pm 0.31^{\mathrm{a}}$ \\
\hline & 遮阴 Shading & $55.8 \pm 1.4^{\mathrm{b}}$ & $0.51 \pm 0.01^{\mathrm{b}}$ & $6 \pm 1^{b}$ & $8.4 \pm 0.6^{b}$ & $67.2 \pm 5.6^{\mathrm{b}}$ & $0.125 \pm 0.006^{\mathrm{b}}$ & $0.33 \pm 0.10^{\mathrm{b}}$ \\
\hline
\end{tabular}

数据后不同字母表示同一作物不同处理间差异显著 $(p<0.05)$ 。

Different letters denote significant differences between treatments $(p<0.05)$.

表5 遮阴对田间豆科作物叶部性状和产量的影响(平均值土标准误差, $n=3$ )

Table 5 Effect of shading on leaves and yields of the legumes in the field experiment (mean $\pm S E, n=3$ )

\begin{tabular}{|c|c|c|c|c|c|c|c|c|}
\hline $\begin{array}{l}\text { 种 } \\
\text { Species }\end{array}$ & $\begin{array}{l}\text { 处理 } \\
\text { Treatment }\end{array}$ & $\begin{array}{l}\text { 单株叶片数 } \\
\text { Leaves quan- } \\
\text { tity }\end{array}$ & $\begin{array}{c}\text { 叶片厚度 } \\
\text { Leaf thickness } \\
(\mathrm{mm})\end{array}$ & $\begin{array}{c}\text { 单片叶面积 } \\
\text { Leaf area } \\
\left(\mathrm{cm}^{2}\right)\end{array}$ & $\begin{array}{c}\text { 叶面积指数 } \\
\text { Leaf area } \\
\text { index }\end{array}$ & $\begin{array}{c}\text { 鲜草产量 } \\
\text { Fresh-grass } \\
\text { yield }\left(\mathrm{kg} \cdot \mathrm{hm}^{-2}\right)\end{array}$ & $\begin{array}{c}\text { 干物质产量 } \\
\text { Dry matter yield } \\
\left(\mathrm{kg} \cdot \mathrm{hm}^{-2}\right)\end{array}$ & $\begin{array}{c}\text { 干物质积累效率 } \\
\text { Efficiency of dry } \\
\text { matter accumulation } \\
\left(\mathrm{kg} \cdot \mathrm{hm}^{-2} \cdot \mathrm{d}^{-1}\right)\end{array}$ \\
\hline \multirow{2}{*}{$\begin{array}{l}\text { 绿豆 } \\
\text { Vigna radiata }\end{array}$} & 对照 CK & $67 \pm 5^{\mathrm{a}}$ & $0.34 \pm 0.03^{\mathrm{a}}$ & $58.3 \pm 3.3^{\mathrm{a}}$ & $3.91 \pm 0.22^{\mathrm{a}}$ & $12070 \pm 483^{\mathrm{a}}$ & $2076 \pm 83^{\mathrm{a}}$ & $49.4 \pm 2.2^{\mathrm{a}}$ \\
\hline & 遮阴 Shading & $41 \pm 4^{\mathrm{b}}$ & $0.26 \pm 0.01^{\mathrm{b}}$ & $29.7 \pm 1.7^{\mathrm{b}}$ & $1.22 \pm 0.11^{\mathrm{b}}$ & $8503 \pm 344^{b}$ & $1386 \pm 56^{\mathrm{b}}$ & $35.5 \pm 1.4^{\mathrm{b}}$ \\
\hline \multirow{2}{*}{$\begin{array}{l}\text { 乌豇豆 } \\
\text { Vigna cylindrica }\end{array}$} & 对照 CK & $91 \pm 6^{\mathrm{a}}$ & $0.29 \pm 0.02^{\mathrm{a}}$ & $51.2 \pm 2.9^{\mathrm{a}}$ & $4.66 \pm 0.26^{\mathrm{a}}$ & $15411 \pm 614^{\mathrm{a}}$ & $2435 \pm 97^{\mathrm{a}}$ & $60.9 \pm 2.7^{\mathrm{a}}$ \\
\hline & 遮阴 Shading & $51 \pm 2^{b}$ & $0.21 \pm 0.01^{b}$ & $39.4 \pm 1.4^{b}$ & $2.01 \pm 0.13^{b}$ & $11562 \pm 451^{b}$ & $1769 \pm 69^{b}$ & $44.2 \pm 2.0^{\mathrm{b}}$ \\
\hline \multirow{2}{*}{$\begin{array}{l}\text { 大猪屎豆 } \\
\text { Crotalaria assamica }\end{array}$} & 对照 CK & $89 \pm 6^{\mathrm{a}}$ & $0.33 \pm 0.02^{\mathrm{a}}$ & $36.0 \pm 1.9^{\mathrm{a}}$ & $3.20 \pm 0.18^{\mathrm{a}}$ & $18135 \pm 785^{\mathrm{a}}$ & $2956 \pm 128^{\mathrm{a}}$ & $46.2 \pm 3.8^{\mathrm{a}}$ \\
\hline & 遮阴 Shading & $39 \pm 3^{b}$ & $0.16 \pm 0.01^{\mathrm{b}}$ & $12.9 \pm 1.0^{\mathrm{b}}$ & $0.50 \pm 0.10^{\mathrm{b}}$ & $5487 \pm 308^{b}$ & $856 \pm 48^{\mathrm{b}}$ & $13.4 \pm 1.2^{\mathrm{b}}$ \\
\hline \multirow{2}{*}{$\begin{array}{l}\text { 望江南 } \\
\text { Senna occidentalis }\end{array}$} & 对照 CK & $168 \pm 9^{\mathrm{a}}$ & $0.17 \pm 0.01^{\mathrm{a}}$ & $23.6 \pm 1.6^{\mathrm{a}}$ & $3.96 \pm 0.19^{\mathrm{a}}$ & $19379 \pm 706^{\mathrm{a}}$ & $4089 \pm 149^{\mathrm{a}}$ & $54.5 \pm 3.8^{\mathrm{a}}$ \\
\hline & 遮阴 Shading & $82 \pm 8^{b}$ & $0.13 \pm 0.01^{\mathrm{b}}$ & $14.0 \pm 1.5^{\mathrm{b}}$ & $1.15 \pm 0.15^{\mathrm{b}}$ & $7020 \pm 322^{b}$ & $1397 \pm 64^{b}$ & $15.9 \pm 1.0^{\mathrm{b}}$ \\
\hline
\end{tabular}

数据后不同字母表示同一作物不同处理间差异显著 $(p<0.05)$ 。

Different letters denote significant differences between treatments $(p<0.05)$.

表6 遮阴对田间豆科作物生殖生长的影响(平均值土标准误差, $n=3$ )

Table 6 Effect of shading on reproductive growth of the legumes in the field experiment (mean $\pm S E, n=3$ )

\begin{tabular}{|c|c|c|c|c|c|c|c|c|c|}
\hline $\begin{array}{l}\text { 种 } \\
\text { Species }\end{array}$ & $\begin{array}{l}\text { 处理 } \\
\text { Treatment }\end{array}$ & $\begin{array}{l}\text { 出苗-初花天数 } \\
\text { Emerging to } \\
\text { first bloom (d) }\end{array}$ & $\begin{array}{l}\text { 花期持续天数 } \\
\text { Flowering con- } \\
\text { tinuously time (d) }\end{array}$ & $\begin{array}{l}\text { 全生育期 } \\
\text { Whole growth } \\
\text { stage }(\mathrm{d})\end{array}$ & $\begin{array}{c}\text { 每株开花数 } \\
\text { Flowers per } \\
\text { plant }\end{array}$ & $\begin{array}{c}\text { 每株荚数 } \\
\text { Pods per } \\
\text { plant }\end{array}$ & $\begin{array}{c}\text { 荚长 } \\
\text { Pod length } \\
(\mathrm{cm})\end{array}$ & $\begin{array}{c}\text { 千粒重 } \\
\text { Thousand-grain } \\
\text { weight }(\mathrm{g})\end{array}$ & $\begin{array}{l}\text { 种子产量 } \\
\text { Seed yield } \\
\left(\mathrm{kg} \cdot \mathrm{hm}^{-2}\right)\end{array}$ \\
\hline \multirow{2}{*}{$\begin{array}{l}\text { 绿豆 } \\
\text { Vigna radiata }\end{array}$} & 对照 CK & $42 \pm 0^{\mathrm{a}}$ & $21 \pm 1^{\mathrm{a}}$ & $68 \pm 1^{\mathrm{a}}$ & $35 \pm 2^{\mathrm{a}}$ & $33 \pm 2^{\mathrm{a}}$ & $9.2 \pm 0.7^{\mathrm{a}}$ & $43.2 \pm 2.0^{\mathrm{a}}$ & $757 \pm 43^{\mathrm{a}}$ \\
\hline & 遮阴 Shading & $39 \pm 1^{b}$ & $12 \pm 0^{\mathrm{b}}$ & $60 \pm 1^{b}$ & $9 \pm 1^{b}$ & $7 \pm 1^{b}$ & $6.9 \pm 0.5^{b}$ & $34.6 \pm 1.6^{\mathrm{b}}$ & $125 \pm 12^{\mathrm{b}}$ \\
\hline 乌豇豆 & 对照 CK & $40 \pm 0^{\mathrm{a}}$ & $37 \pm 1^{\mathrm{a}}$ & $83 \pm 1^{a}$ & $34 \pm 2^{\mathrm{a}}$ & $30 \pm 2^{\mathrm{a}}$ & $15.8 \pm 0.6^{\mathrm{a}}$ & $99.5 \pm 2.2^{\mathrm{a}}$ & $1595 \pm 87^{\mathrm{a}}$ \\
\hline $\begin{array}{l}\text { Vigna } \\
\text { cylindrica }\end{array}$ & 遮阴 Shading & $40 \pm 1^{\mathrm{a}}$ & $33 \pm 0^{\mathrm{b}}$ & $82 \pm 1^{\mathrm{a}}$ & $11 \pm 1^{\mathrm{b}}$ & $8 \pm 1^{\mathrm{b}}$ & $13.1 \pm 0.5^{\mathrm{b}}$ & $90.9 \pm 1.5^{\mathrm{b}}$ & $341 \pm 26^{\mathrm{b}}$ \\
\hline 大猪屎豆 & 对照 CK & $64 \pm 0$ & $27 \pm 1$ & $107 \pm 1$ & $238 \pm 15$ & $225 \pm 15$ & $5.0 \pm 0.3$ & $16.2 \pm 0.6$ & $969 \pm 61$ \\
\hline $\begin{array}{l}\text { Crotalaria } \\
\text { assamica }\end{array}$ & 遮阴 Shading & - & - & - & - & - & - & - & - \\
\hline 望江南 & 对照 CK & $75 \pm 0^{\mathrm{b}}$ & $36 \pm 0^{\mathrm{a}}$ & $123 \pm 0$ & $280 \pm 14^{\mathrm{a}}$ & $264 \pm 14$ & $11.7 \pm 0.9$ & $17.7 \pm 0.9$ & $2326 \pm 105$ \\
\hline $\begin{array}{l}\text { Senna } \\
\text { occidentalis }\end{array}$ & 遮阴 Shading & $88 \pm 1^{\mathrm{a}}$ & $11 \pm 0^{\mathrm{b}}$ & - & $5 \pm 1^{\mathrm{b}}$ & - & - & - & - \\
\hline
\end{tabular}

数据后不同字母表示同一作物不同处理间差异显著 $(p<0.05)$ 。

Different letters denote significant differences between treatments $(p<0.05)$.

开花, 没有种子, 不能完成生活史; 望江南推迟 13 天开花，花期持续天数缩短 25 天，开花数很少，花
朵变小, 花器发育不良, 不能结荚成熟, 没有种子, 不能完成生活史。 


\section{3 讨论和结论}

根据资料(张建新等, 2013)分析：正常光照下， 光饱和点 $(L S P)<460 \mu \mathrm{mol} \cdot \mathrm{m}^{-2} \cdot \mathrm{s}^{-1}$ 、光补偿点 $(L C P)$ $<46 \mu \mathrm{mol} \cdot \mathrm{m}^{-2} \cdot \mathrm{s}^{-1}$ 的是阴性植物或耐阴植物, 否则就 是阳性植物或喜光植物。本实验供试作物对照(全 光)处理的 $L S P$ 均在 $1000 \mu \mathrm{mol} \cdot \mathrm{m}^{-2} \cdot \mathrm{s}^{-1}$ 以上, 大猪屎 豆和望江南的 $L C P$ 在 $46 \mu \mathrm{mol} \cdot \mathrm{m}^{-2} \cdot \mathrm{s}^{-1}$ 以上, 而绿豆和 乌豇豆的 $L C P$ 在 $46 \mu \mathrm{mol} \cdot \mathrm{m}^{-2} \cdot \mathrm{s}^{-1}$ 以下, 说明大猪屎豆 和望江南属于典型的阳性植物; 而绿豆和乌豇豆由 于 $L S P$ 符合阳性植物的标准而 $L C P$ 符合阴性植物的 标准, 说明其既不是典型的阳性植物也不是典型的 阴性植物, 实际耐阴能力介于阳性植物和阴性植物 之间, 同时也说明其对光的适应范围较宽, 既能在 全光条件下生长良好 $(L S P$ 较高, 说明在强光下也不 易受到光抑制), 也能适应一定的弱光环境 ( $L C P$ 较 低, 说明其在弱光下也能维持光合产物的净积累, 有一定的耐阴能力)。遮阴后, 乌豇豆、绿豆和望江 南的 $L S P$ 和 $L C P$ 均显著降低, 说明这些作物的光合 机构在弱光下进行了适当的调整以适应弱光环境, 而大猪尿豆 $L C P$ 改变不明显; 在弱光下, 乌豇豆的 5 个光合特征参数 $\left(P_{\mathrm{nmax}}, L S P, L C P, R_{\mathrm{d}}, A Q Y\right)$ 均向耐阴 植物变化, 绿豆有 4 个参数、望江南有 3 个参数向耐 阴植物变化, 而大猪屎豆仅 2 个参数发生明显变化。 据此可初步得出供试作物对弱光的适应能力顺序是 乌豇豆>绿豆>望江南>大猪屎豆。

影响植物光合能力的生理因素包括气孔因素和 非气孔因素。气孔因素包括 $G_{\mathrm{s}} 、 T_{\mathrm{r}}$ 和 $C_{\mathrm{i}}, G_{\mathrm{s}}$ 较高时叶 片气孔开度较大, 通气能力较强, 单位时间内 $\mathrm{CO}_{2}$ 分子进入量较多, $\mathrm{H}_{2} \mathrm{O}$ 分子反向逸出量也多, $G_{\mathrm{s}}$ 升高 常伴随着 $T_{\mathrm{r}}$ 提高, 所以 $P_{\mathrm{n}}$ 一般都与 $G_{\mathrm{s}}$ 和 $T_{\mathrm{r}}$ 有显著正 相关关系(陈暗等, 2006; 姜武等, 2007; 刘玉梅等, 2010)。本实验的供试作物也是如此, 根据表 2 得出, $P_{\mathrm{n}}$ 与 $G_{\mathrm{s}} 、 T_{\mathrm{r}}$ 的相关系数分别达 $0.8640(p<0.01)$ 和 $0.8957(p<0.01)$, 说明遮阴后作物光合能力的下降 与气孔因素密切相关。而 $P_{\mathrm{n}}$ 与 $C_{\mathrm{i}}$ 的关系比较复杂, 如果 $P_{\mathrm{n}}$ 与 $C_{\mathrm{i}}$ 正相关, 说明气孔因素占主导地位; 如 果 $P_{\mathrm{n}}$ 下降的同时还伴随着 $C_{\mathrm{i}}$ 的增加(负相关), 则说 明非气孔因素也发挥了相当大的作用(Farquhar \& Sharkey, 1982), 本实验中(表2), 供试作物在遮阴后 $P_{\mathrm{n}}$ 下降的同时还伴随着 $C_{\mathrm{i}}$ 的显著增加, 且 $P_{\mathrm{n}}$ 与 $C_{\mathrm{i}}$ 的 相关系数为 $-0.8775(p<0.01)$, 这说明 $P_{\mathrm{n}}$ 下降还与 非气孔因素有关。光合生理的非气孔因素包括光合
色素和光合酶, 以及其他与物质迁移和能量转换相 关的生理生化机制(陈显等, 2006; 张建新等, 2013)。 光合色素主要包括Chl a、Chl b 和 Car, $\mathrm{Chl}$ a的主要功 能是进行光化学反应, $\mathrm{Chl} b$ 的功能主要是收集光能, 在弱光下, $\mathrm{Chl} b$ 的含量显得更重要, 因而 $\mathrm{Chl} a / b$ 值 是植物耐阴性能的重要指标之一, Chl a/b 降低有利 于充分利用弱光, 这是一般耐阴植物适应弱光环境 的正常反应(陈显等, 2006; 姜武等, 2007); Car的功 能主要是在强光条件下耗散过剩的光能, 减少紫外 线的伤害, 防止叶绿素被破坏(陈显等, 2006; 姜武 等, 2007), 在弱光下, Car变得不重要, 因而, 减少 Car含量有利于节约资源; 植物体内的光合酶以 RuBPCase最为关键，其总量占叶绿体可溶蛋白的 50\%-60\% (王学奎, 2006), 因而RuBPCase活性是光 合能力的重要指标(Stitt \& Schulze, 1994; 王学奎, 2006)。本实验中, 乌豇豆在遮阴后, 叶片中的 Chl a、 $\mathrm{Chl} \mathrm{b}$ 和Chl总量显著增加, $\mathrm{Chl} \mathrm{a} / \mathrm{b}$ 和Car含量在遮阴 后显著降低，表现出较强的耐阴性能; 绿豆除Car 外, Chl指标的变化趋势与乌豇豆相似, 表现出中等 的耐阴性能; 望江南在遮阴后虽然Chl含量显著增 加但Chl a/b 和Car变化不明显, 耐阴能力比前 2 种作 物差一些; 遮阴导致大猪屁豆的光合产物供应不 足, 妨碍了叶绿素的合成, 使其光合能力和生长发 育严重受阻。供试的各种豆科作物在遮阴后, RuBPCase活性均显著降低, RuBPCase活性与 $P_{\mathrm{n}}$ 呈 极显著正相关关系 $(r=0.9224, p<0.01)$, 这些指标 都说明非气孔因素在豆科作物耐阴机制中也占有重 要地位。

据资料分析(张建新等, 2013), 当Chl总量> 3.8 $\mathrm{mg} \cdot \mathrm{g}^{-1} 、 \mathrm{Chl} \mathrm{a} / \mathrm{b}$ 值 $<3.2$ 时, 可以判断为阴性植物。表 3 中, 弱光下的乌豇豆和绿豆符合这个标准, 表明弱 光下这 2 种作物的光合机构已经向阴性植物转变。

遮阴后的豆科作物在植株形态上的变化与其他 植物有类似的趋势(陈显等, 2006; 姜武等, 2007; 刘 玉梅等, 2010; 张云等, 2014), 包括茎变细, 侧枝数 减少, 生物量和干物质积累效率降低, 根冠比降低, 叶片变小变薄, 叶片数和叶面积指数减少等。叶片 变薄可增大比叶面积, 使有限的营养物质创造出更 大的受光面积以利吸收更多光能, 这是植物对弱光 环境的正常反应。在以上各种植株形态指标中, 对 干物质产量影响最大的是叶面积指数 $(L A I)$, 本实验 中(表5), $L A I$ 与干物质产量的相关系数 $r=0.7746(p$

www.plant-ecology.com 
$<0.05)$, 与干物质积累效率的相关系数 $r=0.9189$ $(p<0.01)$, 说明 $L A I$ 决定了作物的群体光合能力。本 试验还揭示, 弱光下的豆科作物在根系不发达的同 时, 根瘤的生长受到更严重的抑制(减少至1/6-1/8), 此现象及其机理鲜有报道, 有待进一步研究, 建议 从物质与能量再分配以及信息传导等方面入手, 比 如: 豆科作物在弱光下, 由于蒸腾速率降低, 根系 吸收水分的压力减轻, 在光合产物供应不足的情况 下，营养物质优先分配给地上部以保证茎叶的生长, 在根系获得的营养物质数量减少、根系活力降低的 情况下，根瘤量也随之大量减少。

弱光胁迫对供试作物的生殖生长有显著的抑制 作用, 使其开花数量显著减少, 种子产量显著降低 甚至绝收。但不同作物有不同的表现：乌豇豆开花 与成熟基本正常, 但花期缩短; 绿豆开花提前、花 期缩短并提前成熟; 望江南花而不实; 大猪屎豆不 开花。

从鲜草产量(表5)看, 全光下以望江南为最高, 望江南 $>$ 大猪屎豆 $>$ 乌豇豆 $>$ 绿豆; 而弱光下是乌豇 豆最高, 乌豇豆>绿豆>望江南>大猪屎豆; 从种子 产量(表6)看, 全光下是望江南最高, 而弱光下以乌 豇豆为最高, 这说明, 在供试的 4 种豆科作物中, 乌 豇豆耐阴能力最强。

根据供试作物的光合特性和生长发育对弱光的 响应特征, 以及遮阴后产量的变化, 得出4种供试豆 科作物对弱光的适应能力排序是：乌豇豆 $>$ 绿豆 $>$ 望 江南>大猪屎豆。乌豇豆和绿豆株型不高, 生育期 短, 弱光下仍能维持较高的鲜草产量和干物质积累 效率, 能完成生活史, 适宜与玉米、棉花、茶果、疏 林及其他主作物间作套种; 望江南和大猪屎豆喜 光, 遮阴后其生长发育严重受阻, 鲜草产量和干物 质积累效率显著降低且不能结籽, 不宜作为套种 作物。

基金项目 国家公益性行业(农业)科研专项经费项 目(201103005), 国家农作物种质资源平台运行服务 项目 (2012-019)，作物种质资源保护和利用项目 (NB2013-2130135-34)。

致谢 感谢安徽科技学院周毅博士在光合参数测定 中给予的帮助!

\section{参考文献}

Awal MA, Koshi H, Ikeda T (2006). Radiation interception and use by maize/peanut intercrop canopy. Agricultural and
Forest Meteorology, 139, 74-83.

Bloor JMG, Grubb PJ (2003). Growth and mortality in high and low light: Trends among 15 shade-tolerant tropical rain forest tree species. Journal of Ecology, 91, 77-85.

Chen Y, Yang ZM, Li ZH (2006). Review of studies on turfgrass shade-tolerance. Chinese Journal of Grassland, 28(3), 71-76. (in Chinese with English abstract) [陈显, 杨 志民, 李志华 (2006). 草坪草耐荫性研究进展. 中国草 地学报, 28(3), 71-76.]

Farquhar GD, Sharkey TD (1982). Stomatal conductance and photosynthesis. Annual Review of Plant Physiology, 33, 317-345.

Harrison WG, Platt T (1986). Photosynthesis-irradiance relationships in polar and temperate phytoplankton populations. Polar Biology, 5, 153-164.

Jiang W, Jiang WB, Li ZG (2007). Advance of researches on germplasm differences and genetic expression of photosynthetic traits in horticultural crops. Nonwood Forest Research, 25(4), 102-108. (in Chinese with English abstract) [姜武, 姜卫兵, 李志国 (2007). 园艺作物光合性状种 质差异及遗传表现研究进展. 经济林研究, 25(4), 102-108.]

Knox J, Morris J, Hess T (2010). Identifying future risks to UK agricultural crop production: Putting climate change in context. Outlook on Agriculture, 39, 249-256.

Li GH, Wan YS, Liu FZ, Zhang K (2014). Photosynthetic characteristics in different peanut cultivars under conditions of drought and re-watering at seedling stage. Chinese Journal of Plant Ecology, 38, 729-739. (in Chinese with English abstract) [厉广辉, 万勇善, 刘风珍, 张昆 (2014). 苗期干旱及复水条件下不同花生品种的光合特 性. 植物生态学报, 38, 729-739.]

Li L, Tang CX, Rengel Z, Zhang FS (2003). Chickpea facilitates phosphorus uptake by intercropped wheat from an organic phosphorus source. Plant and Soil, 248, 297-303.

Li L, Tang C, Rengel Z, Zhang FS (2004a). Calcium, magnesium and microelement uptake as affected by phosphorus sources and interspecific root interactions between wheat and chickpea. Plant and Soil, 261, 29-37.

Li SM, Li L, Zhang FS, Tang C (2004b). Acid phosphatase role in chickpea/maize intercropping. Annals of Botany, 94, 297-303.

Liu YM, Ai XZ, Yu XC (2010). Effects of ALA on photosynthesis of cucumber seedlings under suboptimal temperature and light intensity. Acta Horticulturae Sinica, 37, 65-71. (in Chinese with English abstract) [刘玉梅, 艾希 珍, 于贤昌 (2010). 5-氨基乙酰丙酸对亚适宜温光条件 下黄瓜幼苗光合特性的影响. 园艺学报, 37, 65-71.]

Qi J, Shi SL, Xu CL, Yan WH, Zhang XJ (2013). A comparison of photosynthesis responses to light of four Elymus species. Acta Prataculturae Sinica, 22(6), 100-107. (in 
Chinese with English abstract) [祁娟, 师尚礼, 徐长林, 问伟红, 张小娇 (2013). 4种披碱草属植物光合作用光 响应特性的比较. 草业学报, 22(6), 100-107.]

Stitt M, Schulze D (1994). Does Rubisco control the rate of photosynthesis and plant growth? An exercise in molecular ecophysiology. Plant, Cell \& Environment, 17, 465-487.

Tanwar SPS, Rao SS, Regar PL, Datt S, Kumar P, Jodha BS, Santra P, Kumar R, Ram R (2014). Improving water and land use efficiency of fallow-wheat system in shallow Lithic Calciorthid soils of arid region: Introduction of bed planting and rainy season sorghum-legume intercropping. Soil and Tillage Research, 138, 44-55.

Wang XK (2006). Principles and Techniques of Plant Physiological Biochemical Experiment. Higher Education Press, Beijing. 134-139, 195-201. (in Chinese) [王学奎 (2006). 植物生理生化实验原理和技术. 高等教育出版社, 北 京. 134-139, 195-201.]

Wen MX, Shi XJ, Nie ZP, Liu WF, Zhou XB (2011). Effect of summer green manure in Pankan tangerine orchard. Journal of Fruit Science, 28, 1077-1081. (in Chinese with English abstract) [温明霞, 石孝均, 聂振朋, 刘文峰, 周 金斌 (2011). 椪柑果园种植夏季绿肥的效应. 果树学 报, 28, 1077-1081.]

Wu H, Dai HF, Zhang JS, Jiao XL, Liu C, Shi JY, Fan ZC, Aliyan R (2014). Responses of photosynthetic characteristics to low temperature stress and recovery treatment in cotton seedling leaves. Chinese Journal of Plant Ecology,
38, 1124-1134. (in Chinese with English abstract) [武辉, 戴海芳, 张巨松, 焦晓玲, 刘翠, 石俊毅, 范志超, 阿丽 艳·肉孜 (2014). 棉花幼苗叶片光合特性对低温胁迫及 恢复处理的响应. 植物生态学报, 38, 1124-1134.]

Zhang CP, Meng P, Li JZ, Wan XC (2014). Interactive effects of soil acidification and phosphorus deficiency on photosynthetic characteristics and growth in Juglans regia seedlings. Chinese Journal of Plant Ecology, 38, 1345-1355. (in Chinese with English abstract) [张翠萍, 孟平, 李建 中, 万贤崇 (2014). 磷元素和土壤酸化交互作用对核桃 幼苗光合特性的影响. 植物生态学报, 38, 1345-1355.]

Zhang JX, Yan Y, Fang YM (2013). Effect of shading on growth and photosynthetic characteristics of Clerodendrum bungei. Journal of Plant Resources and Environment, 22(1), 88-93. (in Chinese with English abstract) [张 建新, 颜传, 方炎明 (2013). 遮光对臭告丹生长和光合 特性的影响. 植物资源与环境学报, 22(1), 88-93.]

Zhang Y, Xia GH, Ma K, Dai GY, Dai YC, Yan CX (2014). Effects of shade on photosynthetic characteristics and chlorophyll fluorescence of Ardisia violacea. Chinese Journal of Applied Ecology, 25, 1940-1948. (in Chinese with English abstract) [张云, 夏国华, 马凯, 李根有, 代 英超, 严彩霞 (2014). 遮阴对堇叶紫金牛光合特性和叶 绿素荧光参数的影响. 应用生态学报, 25, 1940-1948.]

责任编委: 储诚进 责任编辑: 李 敏 